\title{
Racism, racial life events and mental ill health
}

\section{Dinesh Bhugra and Oyedeji Ayonrinde}

The association of life events with the onset of various psychiatric disorders is well-known. The body of evidence has highlighted the impact of negative or positive life events on the genesis of common mental disorders, especially depression. These findings have been replicated across different cultures, although the impact of different life events varies between cultures. In addition, the roles of chronic difficulties (defined in Life events and psychiatric disorders, below) and resulting ongoing chronic stress have been shown to contribute to vulnerability to certain mental illnesses. However, data on the impact of life events, especially those perceived as racial, on members of minority ethnic groups are rather sparse. The questions that need to be addressed concern the perception of life events as racial, the role of pervasive and perceived institutional and individual racism, and chronic difficulties.

In this review, we aim to draw the clinician's attention to some of these key factors, which may precipitate or perpetuate ongoing psychiatric problems.

\section{Race and racism}

Until the middle of the 20th century race was used as a Darwinian tool to emphasise that Blacks were inferior and savage compared with the Whites. This was accepted as a biological fact in the scientific community, thereby giving it a scientific legitimacy. Racism is of several types, both at individual and institutional levels.
The term 'race' has three usages: biological, common and political (Fuller \& Toon, 1988). In biological usage, 'race' refers to the genetic separateness of different groups: each 'racial' group has an average genetic make-up that differs in some forms from the genetic make-up of other groups. However, genetic differences within each race are so wide that two individuals within the same racial group may have differences greater than the average differences between two different groups. Races are not separate, and the boundaries between them are arbitrary. In medicine, race is often used as a category that allows clinicians to link specific illnesses with particular races, for example cystic fibrosis and some White groups. Such distinctions can legitimise racist thinking.

In common usage, race has become synonymous with physical appearance, and skin colour is given undue importance.

The political use of the term allows the majority of society to consolidate power and minority groups to politicise their identity.

In its Lexicon of Cross-Cultural Terms in Mental Health, the World Health Organization (1997) has defined racism, prejudice and ethnocentrism as follows. Racism is the belief that there is an inherent connection between perceived hereditary and cultural traits, and that some groups are biologically superior to others. Prejudice is the negative attitudinal and emotional set against an individual or group based on selected social or cultural characteristics. Ethnocentrism is the overvaluing of one's own culture in relation to other cultures, so that biased judgements are made regarding what is good, correct, beautiful, moral, normal, sane or rational. Individual views on racism differ from institutional

Dinesh Bhugra is a reader in cultural psychiatry and heads the Section of Cultural Psychiatry at the Institute of Psychiatry (De Crespigny Park, London SE5 8AF). His research interests include the influence of culture on mental illness, spirituality, sexual dysfunction and sexual deviation. Oyedeji Ayonrinde is a specialist registrar in the South London and Maudsley NHS Trust. His research interests include mental disorders, especially affective disorders, common in West African groups and their management. 
racism, which is a collective view within an organisation that is deeply embedded in the way in which it functions. While most professionals may not favour the theory of (genetic) inherited psychological inferiority, it remains a standard way of thinking among the lay public, where individual traits are described away as being "in the blood" (Thomas \& Sillen, 1991).

'Ethnicity' is often used both to remove the pejorative implications of 'race' and in recognition that different races may share a similar culture. Essentially, ethnicity refers to a psychological sense of belonging, which will often be cemented by similarities in physical appearance or social behaviour (MacLachlan, 1997).

The MacPherson Report (MacPherson, 1999) defined institutional racism as

"the collective failure of an organisation to provide an appropriate and professional service to people because of their colour, culture or ethnic origin. It can be seen or detected in processes, attitudes and behaviour which amount to discrimination through unwitting prejudice, ignorance, thoughtlessness, and racist stereotyping which disadvantage minority ethnic people."

A key difficulty with such a definition is that it argues for detecting flaws in the processes by which an organisation (as a living organism) works, and it is not always clear what these processes are, who identifies the flaws and who should deal with them. Subjective experiences or interpretations of racism are even more difficult to define, as some are directly linked with an individual's personality traits, previous experiences and support systems (social and economic).

Bhugra \& Bhui (1999) have previously argued that subjugation of ethnic minorities by the majority using historical, social, biological and economic factors is common throughout history. There is no doubt that racism and ideas pertaining to it date back to the pre-Christian era. In $100 \mathrm{BC}$, Cicero advised Atticus not to buy slaves from Britain as they were stupid, lazy and incapable of learning. However, the ideology behind racism is based on the wish to maintain the status quo and on the belief in the superiority of one group or another on purely racial or biological grounds. Race is a taxonomic concept of limited usefulness, and over the past 30 years it has started to give way to ethnicity or cultural groupings, which are very much more amorphous. Racism can be seen as ideology, as practice and as social structure.

Racism must be distinguished from racial discrimination. The former is restricted to discourses about grouping human populations into racial stocks (which may lead to ethnocentrism). The latter, however, is to do with actual behaviours. Racism

\section{Box 1 Types of racism}

Dominative Hatred turns into actions

Aversive Individual feels superior but is unable to act

Regressive Individual's views on racism result in regressive behaviour

Pre-reflecting gut racism Fear of strangers

Post-reflecting gut racism Rationalisation, justification of fear of strangers

Cultural Rejection, denigration of leisure, social customs

Institutional Organisations' views on perceived inferiority

Paternalistic Majority 'know' what is good for minority

Colour-blind Acceptance of differences is seen as culturally divisive

New racism Hidden in 'individualism': positive action is objected to and existent racism criticised on the grounds of present achievements of the group

takes many forms, some of which are described in Box 1 .

It must be emphasised that racism is not a static phenomenon. Furthermore, it should also be distinguished from racialism, which is the acting out of racial prejudice by one individual towards another. Racism includes the use of beliefs or practices to justify and maintain inequity, exclusion or domination. An interesting development is the use of 'colour-blindness' as a form of racism. When the 'colour-blind' look at subordinate groups of people of different skin colour they do not see them as people with their own history, culture, spiritual and socio-economic realities. Racism can also exacerbate the health effects of poverty. Moore (2000) suggests that the psychology of colonialism and the control of information, communications and liberty are important factors in the development of racism. A dominative racist is one who acts on bigotry, whereas the aversive racist exhibits dislike and avoidance. There are those who do not reveal their racist tendencies except as unconscious aspects of mass behaviour (Kovel, 1984). The hatred of the 'outgroup' (defined in Racial life events, below) and authoritarianism also contribute to the maintenance of the status quo.

Psychiatry reflects dominant social values and can both be oppressive and be seen to be oppressive when individuals are detained against their will. This leads to a sense of alienation, and with time and experience minority individuals may feel further let down. The relationship of racism with 
Box 2 The therapists' self-assessment

Therapists must always be aware of

- their own likes and dislikes

- their own identity, e.g. race, gender, ethnicity, culture, power

- mutual learning from the patient

- the possibility of idealising a particular culture

- the strengths and weaknesses of the patient's culture

- the strengths and weaknesses of their own culture

research and clinical conditions is extremely complex. For clinicians assessing an individual from a different ethnic and cultural background it is essential that they recognise their own views of race (Box 2).

\section{Life events and psychiatric disorders}

The stress diathesis model in the aetiology of mental illness suggest that individuals vulnerable to stress may develop psychiatric disorders under certain circumstances and if adequate social networks or protective factors are not available. It is clear that adverse life events can influence the adaptive potential and mental health of human beings (Rutter, 1985), thereby contributing significantly to psychiatric morbidity. The strongest and most consistent associations have been reported in the aetiology of depression (Brown \& Harris, 1978; Paykel, 1994), although associations have also been found with the onset of psychotic illness (Brown \& Harris, 1978). The association between life events and common mental disorders has likewise been reported in non-Western cultures (Vadher \& Ndetei, 1981; Guereje, 1986; Bebbington et al, 1998). Studies of genetic factors and shared familial life events have suggested that those who are more prone to depression are more likely to perceive events as threatening. Finlay-Jones \& Brown (1981) reported that loss events were particularly important in the onset of depression and dangerous events in the onset of anxiety disorders.

Brown \& Harris (1978) define a 'chronic difficulty' as a problem that has gone on for at least 4 weeks and a 'life event' as a problem resulting as a direct result of any event within a specific period of time.
They observed that in a sample of attenders at a general practice surgery, $38 \%$ of patients with depression had experienced severe life events. Those who had experienced major difficulties (during the previous 2 years) and severe life events comprised $62 \%$ of the depression sample. Chronic major difficulties are likely to be associated with depression, as they are most likely to interfere with cognition and may produce negative cognitions. Social class, economic factors and the help of a close confidant (i.e. social support) provide a degree of buffer for individuals.

The significance of experiences of hopelessness, entrapment, humiliation and defeat in the aetiology of depression has been discussed by Brown (1998). Other depressogenic events include attacks on selfesteem, which force the individual into a subordinate position, undermining his or her sense of rank, attractiveness and value and seemingly blocking escape routes (Gilbert \& Allan, 1998).

It is very likely that similar aetiological models will operate in individuals from ethnic minorities, and clinicians must assess their patients' feelings of self-esteem, sense of entrapment and lack of social support.

\section{Racial life events}

The impact of racial life events on ethnic minorities is varied and wide ranging (Bhugra \& Ayonrinde, 2001). Migration may have significant effects on the individual and the group (see Bhugra \& Cochrane, 2001). Accurate data on the prevalence of racial attacks, violence and racially motivated crime (victimisation, attacks and harassment) are difficult to obtain. There are several reasons for this: the individual may not perceive these attacks as racial and therefore not report them as such; the ethnicity of the perpetrator may not be known; victims may wrongly attribute a racial motive to an incident; further victimisation may prevent reporting; and insufficient evidence may lead to underreporting.

The British Crime Survey (BCS) and police records have different methods of data collection. The BCS records both reported (actual) offences (such as vandalism, burglary, theft, wounding of persons, assault or robbery) and threatened offences; police records, however, show only reported crimes, although they do also note any alleged racial motive or if the investigating officer suspected a racial motive. BCS data deal with individuals over 16 years of age, but police records include all ages. Fitzgerald \& Hale (1996) cite BCS findings that only $2 \%$ of all crimes were considered by victims to be motivated 
by racism, and nearly a quarter of these occurred in inner-city areas.

There are ethnic differences too in reporting of crimes (Commission for Racial Equality, 1999). Bearing in mind the type of crime, method of reporting and delay in reporting, it is important to emphasise that this area remains underresearched.

Chahal \& Julienne (1999) observed that between 43 and $62 \%$ of racial incidents went unreported. Among those that were reported were injuries to the person, harassment, assault and criminal damage to property; incidents related to failure to get a job, a mortgage, or support in schools, hospitals and so on are not even likely to be recorded. In a qualitative study of subjective experiences of racism, they found that subjects described the routine nature of racial incidents in their lives. They also used different pathways for disclosing these events - most of which were in personal or social sectors. Failure to disclose was often due to feelings of shame, inadequacy, hopelessness or mistrust. Only a significant increase in incidents led individuals to disclose to statutory agencies. General practitioners (GPs) were most frequently approached, but the resulting action was often limited (e.g. a GP might write a letter to the housing authorites supporting re-housing, but do nothing else). Thus, even if events are identified, their impact can easily be ignored. Commonly recurring emotional themes in the group were those of anger, stress, depression, heightened arousal and poor sleep.

Racial life events are problems that can be directly attributed to racial behaviour, and they occur in many different domains (Box 3).

Racial difficulties may be defined as ongoing difficulties in an individual's life that can be attributed to racial components and last for more than 1 month. They include problems with housing, employment, social functioning and education.

In addition to stressors that are common to all, minority groups may experience stress resulting from their minority status. These include stressor stimuli (e.g. prejudice, hostility and discrimination);

Box 3 Domains of racial life events

Accommodation

Education

Employment

Finance

Health

Incidents of harassment

Damage to property

Law and welfare

\section{Box 4 Problems related to racism}

Institutional racism

Stereotyping

Rejection

Prejudice

Devaluation of culture

Individual racism

Stereotyping

Rejection

Prejudice

Devaluation of culture

Threats

Attacks

external mediating forces (social support); and internal mediating forces (cognitive factors) that influence the individual's perception of life events. Smith (1985) has suggested the terms 'outgroup' and 'in-group' to describe the situation of minority groups (the out-group) in a majority culture (the in-group). Out-group status results in social isolation, social marginalisation and status inconsistency, which increase the individuals' state of arousal. The incomplete or partial assimilation by minority individuals of the new (host) majority culture and the complete or partial rejection of their own culture may lead to additional stresses.

\section{Racism and mental disorders}

Racism at either the individual or institutional level may lead to a number of problems, some of which are illustrated in Box 4. A sense of status inconsistency may occur, where the individual has two or more distinct and incompatible social statuses (e.g. the individual's social status is contaminated by the status associated with his or her ethnicity). This contradiction in role and status is likely to lead to adjustment difficulties or psychopathology (Smith, 1985). As ethnic minority individuals are more 'visible' among the majority population, their actions take on a symbolic meaning and stereotypes tend to take over. Smith (1985) argues that high visibility, over-observation, lack of anonymity, polarisation and role entrapment are some of the factors that contribute to the stress and chronic difficulties.

Racism is a multi-dimensional phenomenon, and a number of instruments have been developed to measure life events on various axes. An illustrative 
Box 5 Factors mitigating or exacerbating the effect of individual and institutional racism

Mitigating factors

Social support: ethnic group, cultural identity

Exacerbating factors

Stress

Mental illness

Powerlessness

Potentially mitigating or exacerbating factors

Age

Gender

Socio-economic status

example of various domains in some of the instruments is shown in Box 3. Many of these scales have been developed for specific ethnic groups, sometimes for specific ethnic contexts and specific studies. Box 5 lists some factors influencing the effects of racism on the individual. In summary, racism has:

- the ability to create differential social status that results in differential health consequences

- a role in determining differential exposure to risk factors and available resources

- an effect on the individual's psychological well-being and functioning (Herman, 1996; Jackson et al, 1996; Williams, 1996; WilliamMorris, 1996).

Jackson et al (1996) demonstrated that cumulative perceptions of racism and racial discrimination result in poorer mental than physical health. The role of 'locus of control' as an intervening variable for the psychological well-being has to be explored further in the context of ethnic minority groups.

\section{Racial events and psychiatric disorders}

\section{Depression}

The limited data available show that social life events are, like life events in general, significantly associated with depression. Several studies have shown higher rates of depression in minority ethnic groups in the community (Nazroo, 1997; Shaw et al, 1999), with possible explanations including exposure to differential environmental losses, unemployment, poverty and racism. In a study of deliberate self-harm in Asian women, Bhugra et al (1999) reported that nearly a quarter of their sample had experienced racial life events, although a causal association cannot be ascertained from this study.

\section{Anxiety}

Stress models suggest an increase in anxiety in the face of threatening life events. In a study from New Zealand, Pernice \& Brook (1996) reported that racial discrimination was significantly associated with high levels of anxiety among non-White immigrants. They also reported that anxiety levels were surprisingly high among those who spent a lot of leisure time with their own ethnic group. It is possible that these were anxious individuals who were seeking solace in the company of their own ethnic group. Anxiety symptoms have been shown to develop following racial threats (Thompson, 1996; Jones et al, 1996).

\section{Post-traumatic stress disorder}

Increased levels of stress with symptoms akin to post-traumatic stress disorder following experiences of racial discrimination have been reported (Ritsner et al, 1997). Symptoms of hypervigilance, increased arousal, poor concentration, increased levels of frustration, denial, social withdrawal, anxiety and repeated flashbacks have also been reported following a significant racial life event.

\section{Psychosis}

There is anecdotal evidence that suggests links between psychosis and racial events, and racism at an institutional level is said to play a key role in the relationship with compliance and follow-up. The empirical data, however, are inconclusive.

The relationship between racial life events and onset of psychiatric disorders is complex and it is only in recent times that researchers have started working on disentangling these interactions.

\section{Racism and mental distress}

Racism, be it individual or institutional, is likely to act as a chronic stress or in the form of chronic difficulties preventing individuals from progressing. They may feel that although they are capable of achieving more, other people or the system are hindering their progress. Such obstacles may give 
individuals a sense of entrapment and bewilderment, knocking their self-esteem and self-concept. This may also further alienate them from their own ethnic group, especially if their methods of dealing with chronic difficulties are at variance with those of their peers, thereby adding further to their stress.

\section{Conclusion}

Individuals, whatever their ethnic background, interact with their social and cultural milieux and respond to ongoing difficulties or sudden stress in different ways. Actual racial life events, a perception of such events and chronic racism are all likely to precipitate psychiatric disorders. However, the research data remain sparse, and in the studies that have been carried out data-collection methods have sometimes been unclear, making any interpretations and generalisations very difficult.

\section{References}

Bebbington, P., Hamdi, E. \& Ghubash, R. (1998) The Dubai Community Psychiatry Survey IV. Social Psychiatry and Psychiatric Epidemiology, 33, 501-509.

Bhugra, D. \& Ayonrinde, O. (2001) Racial life events and psychiatric morbidity. In Psychiatry in Multicultural Britain (eds D. Bhugra \& R. Cochrane), pp. 91-111. London: Gaskell.

— \& Bhui, K. S. (1999) Racism in psychiatry: paradigm lost, paradigm regained. International Review of Psychiatry, 11, 236-243.

— \& Cochrane, R. (eds) (2001) Psychiatry in Multicultural Britain. London: Gaskell.

— , Baldwin, D., Desai, M., et al (1999) Attempted suicide in West London. II. Psychological Medicine, 29, 1130-1139.

Brown, G. (1998) Genetic and population perspective on life events and depression. Social Psychiatry and Psychiatric Epidemiology, 33, 363-372.

Brown, G. \& Harris, T. (1978) Social Origins of Depression. London: Tavistock.

Chahal, K. \& Julienne, L. (1999) We Can't All Be White. York: York Publishing Services.

Commission for Racial Equality (1999) Ethnic Minorities in Britain. London: CRE.

Finlay-Jones, R. \& Brown, G. (1981) Types of stressful life events and onset of anxiety and depression disorders. Psychological Medicine, 11, 813-815.

Fitzgerald, M. \& Hale, C. (1996) Ethnic Minorities, Victimisation and Racial Harassment. Home Office Research Findings no. 39. London: Home Office.

Fuller, J. H. S. \& Toon, P. D. (1988) Medical Practice in a Multicultural Society. Oxford: Heinemann.

Gilbert, P. \& Allan, S. (1998) The role of defeat and entrapment (arrested flight) in depression: an exploration of an evolutionary view. Psychological Medicine, 28, 585-598.

Guereje, O. (1986) Social factors and depressions in Nigerian women. Acta Psychiatrica Scandinavica, 74, 392-395.

Herman, A. (1996) Toward a conceptualisation of ease in epidemiologic research. Ethnicity and Disease, 6, 7-20.

Jackson, J. S., Brown, T., Williams, D., et al (1996) Racism and the physical and mental health of African Americans. Ethnicity and Disease, 6, 132-147.
Jones, D. R., Harrell, J. P., Morriss-Prather, C., et al (1996) Affective and physiological responses to racism. Ethnicity and Disease, 6, 109-122.

Kovel, J. (1984) White Racism, a Psychohistory. New York: Columbia University Press.

MacLachlan, M. (1997) Culture and Health. Chichester: John Wiley \& Sons.

MacPherson, W. (1999) The Stephen Lawrence Inquiry. Report of an Inquiry by Sir William Macpherson of Cluny. London: Stationery Office.

Moore. L. (2000) Psychiatric contributions to understanding racism. Transcultural Psychiatry, 37, 147-182.

Nazroo, J. (1997) Ethnicity and Mental Health. London: PSI.

Paykel, E. S. (1994) Life events, social support and depression. Acta Psychiatrica Scandinavica Supplementum, 377, 50-58.

Pernice, R. \& Brook, J. (1996) Refugees' and immigrants' mental health. Journal of Social Psychology, 511-519.

Ritsner, M., Ponizoysky, A. \& Ginath, Y. (1997) Changing patterns of distress during the adjustment of recent immigrants. Acta Psychiatrica Scandinavica, 95, 494-499.

Rutter, M. (1985) Resilience in the face of adversity. Protective factors and resistance to psychiatric disorder. British Journal of Psychiatry, 147, 598-611.

Shaw, C. M., Creed, F., Tomenson, B., et al (1999) Prevalence of anxiety and depressive illness and help seeking behaviour in African Caribbeans and white Europeans. BMJ, 318, 302-305.

Smith, E. (1985) Ethnic minorities: life stress, social support and mental health issues. Counseling Psychologist, 4, 537579 .

Thomas, A. \& Sillen, S. (1991) Racism and Psychiatry. New York: Citadel Press.

Thompson, C. E., Neville, H., Weathers, P. C., et al (1996) Cultural mistrust and racism. Journal of College Student Development, 31, 162-168.

Thompson, V. L. (1996) Perceived experiences of racism as stressful life events. Community Mental Health Journal, 32, 223-233.

Vadher, A. \& Ndetei, D. M. (1981) Life events and depression in a Kenyan setting. British Journal of Psychiatry, 139, 134137.

Williams, D. (1996) Racism and health: a research agenda. Ethnicity and Disease, 6, 1-6.

World Health Organization (1997) Lexicon of Cross-Cultural Terms in Mental Health. Geneva: WHO.

William-Morris, R. S. (1996) Racism and children's health Ethnicity and Disease, 6, 69-82.

\section{Multiple choice questions}

1. There is a direct causal relationship between racial life events and:
a schizophrenia
b panic attacks
c depression
d psychoactive substance misuse
e crime.

2. Institutional racism:

$\mathrm{a}$ is defined as a collective organisational failure

b especially involves colour, age, culture, gender and ethnic origin

c involves processes, attitudes and behaviour

$\mathrm{d}$ is always conscious and intentional

$\mathrm{e}$ is discriminatory. 
3. Subjective experience of racism may be influenced by:
a personality traits
b social class
c past experience
d support networks
e mental state.

4. On the epidemiology of racial life events:

a crime records provide an accurate reflection of prevalence

$\mathrm{b}$ a racial motive may be wrongly attributed to events

c failure to disclose incidents has been associated with shame

d increased reporting reflects an actual increase in rates

e evidence suggests that up to $60 \%$ of racial incidents go unreported.
5. In the aetiology of depression, racial stress may act through:

a hopelessness

b entrapment

c humiliation

d defeat

e improved self-esteem.

\begin{tabular}{|c|c|c|c|c|}
\hline \multicolumn{5}{|c|}{ MCQ answers } \\
\hline 1 & 2 & 3 & 4 & 5 \\
\hline a $\mathbf{F}$ & a $\mathrm{T}$ & a $T$ & a $F$ & a $\mathrm{T}$ \\
\hline b $\mathbf{F}$ & b $F$ & b $\mathrm{T}$ & b $T$ & b $T$ \\
\hline c $F$ & c $\mathrm{T}$ & c $\mathrm{T}$ & c $\mathrm{T}$ & c $\quad T$ \\
\hline d F & d F & d $T$ & d F & d $T$ \\
\hline e $\mathbf{F}$ & e $T$ & e $T$ & e $T$ & e $\mathbf{F}$ \\
\hline
\end{tabular}

\section{New from Gaskell}

Peter Liddle

Modern neuroscience has provided us with a foundation for understanding mental disorder in terms of brain dysfunction. Imaging techniques, such as PET and fMRI, have demonstrated graphically the correspondence between patterns of brain activity and patterns of mental activity.

This book draws on evidence from neuroimaging studies, together with evidence from the fields of neuropsychology, cognitive psychology, electrophysiology, neurochemistry and pharmacology, to give a coherent and plausible account of the cerebral processes by which mental symptoms are generated.

It is intended for psychiatrists and psychologists with an interest in the origins of the symptoms thay observe and treat, as well as for neuroscience students and researchers interested in the relationship between findings from the laboratory and the mental disorders that occur in clinical practice. Indeed, it is intended for anyone with a serious interest in how the mind works, and in how mental disorders arise.

\section{July 2001, £40.00, ISBN $190124265 X$}

